\title{
LICHENS OF ŁOMŻA TOWN (PODLASIE, NORTH-EASTERN POLAND)
}

\author{
Anna Matwiejuk, Paulina Chojnowska
} A. Matwiejuk, P. Chojnowska, Institute of Biology, University of Bialystok, Konstanty Ciołkowski 1 J,
15-245 Białystok, Poland, e-mail: matwiej@uwb.edu.pl, pchojnowska@wp.pl

(Received: January 21, 2016. Accepted: March 29, 2016)

\begin{abstract}
This paper presents new distribution stands for 70 species of lichenized town from Łomża town (Podlasie, NE Poland). The investigations in the area of Łomża were carried out in the years 2014-2015, on 34 research stands. Seven species have been put on the Red list of the lichens in Poland (CieśLIŃski et al. 2006), including Rhizocarpon lavatum in critically endangered - CR, Ramalina fastigiata, $R$. fraxinea in the endangered category - EN, in the Ramalina farinacea in the vulnerable category - VU and Evernia prunastri, Hypogymnia tubulosa, Physcia aipolia in the category of near threatened - NT and five have been put under legal protection, two of which are strictly (Ramalina fastigiata, $R$. fraxinea) and three of which are partially protected (Cladonia arbuscula, Hypogymnia tubulosa, Ramalina farinacea). The lichens occur on following substrate types: soil, decaying wood, bark of all trees and shrubs species, boulders, concrete, foundation, mortar, plaster and bryophytes.
\end{abstract}

KEY WORDS: Lichens, distribution, urban area, north-eastern Poland

\section{INTRODUCTION}

Investigations on lichens in Poland have been carried out in a large number of big and small towns, frequently of a health-resort character, situated in the lowlands as well as the mountains. On the territory of Poland lichen biota has been compiled for big cities, such as Lublin (RYDZAK 1953), Radom (CIEŚLIŃSKI 1974), Warszawa (ZimnY \& KuciŃsKA 1974), Kielce (Toвorowicz 1976), Kraków (KiszKa 1977), Słupsk (ŚPIEWAKOWSKI \& IzYdorek 1981), Gdańsk, Sopot, Gdynia (FaŁtynowicz et al. 1991), Rzeszów (PusTELNIAK 1991), Przemyśl (KIszKa 1999). However, data concerning small towns populated by up to a few thousand inhabitants are not numerous, e.g. for Muszyna, Wisła (RYDzAK 1956a), Wołczyn (RYDZAK 1956 b), Duszniki Zdrój, Polanica Zdrój (RYDZaK 1959), Limanowa (JaGięŁo 1983), Drezdenko (LipNICKI 1984). No comprehensive studies on lichens have been carried out in north-eastern Poland except for Białowieża (Rydzak 1957, Matwiejuk 2011), Olsztyn (Kubiak 2005), Białystok (MaTwiejuk 2007), Ciechanowiec (Matwiejuk \& Kolanko 2007), Mielnik (Matwiejuk 2008), Boćki (Matwiejuk 2009a), Drohiczyn (Matwiejuk 2009b), Narew (Matwiejuk \& Korobkiewicz 2012 a), Ełk (Matwiejuk \& Wójtowicz
2013), Supraśl (Matwiejuk 2015). This area, which constitutes over $11 \%$ of Poland, is in many ways greatly differentiated from other parts of Poland. Its idiosyncratic geological structure, features of climate and the plant cover, as well as the history, ethnographic background and economic relations of the region determine its separate nature (KONDRACKI 1972).

This inspired lichenological investigations in Łomża, the fourth largest urban centre in north-east Poland after Białystok, Olsztyn, Suwałki.

The aim of the study was to present documentation illustrating biodiversity of lichen biota in the area of Łomża, taking into consideration the habitat conditions of species.

\section{STUDY AREA}

City towering over Narew - such a picturesque view appears in the eyes of travelers who enter here from the Piątnica. Łomża buildings occupied high nadnarwiańska slope and location is, of course, is a great asset. Over 1,000 years ago, when in the area assumed the first settlement from the picturesque position were more important considerations defensive. Castle, which is still acting on the imagination of the 
Table 1. The emissions in Łomża in 2014

\begin{tabular}{ccccc}
\hline & \multicolumn{4}{c}{ Emissions in 2014 $\left(\mu \mathrm{g} / \mathrm{m}^{3}\right)$} \\
\cline { 2 - 5 } & $\mathrm{SO}_{2}$ & $\mathrm{NO}_{2}$ & dust PM 10 & dust PM 2.5 \\
\hline Total & 6.5 & 15.9 & 29.5 & 28.0 \\
\hline
\end{tabular}

Data source: WIOŚ Białystok.

remains can be found near the present town, guarded the trade route and crossing on Narew. Since 1418 Łomża enjoys the city charter. Her fate was kind to the sixteenth century. Two more centuries experienced her mercilessly, just as World War II, when he destroyed almost $80 \%$ of the buildings. Nevertheless, the city has retained interesting sights, which is worth looking at closely. The most important object is a sixteenth-century cathedral. Certainly Łomża would not be what it is, if it were not Narew river, which belongs to the natural pearls of north-eastern Polish. Her spring flood waters can admire, without leaving even outside buildings (ZuBKowicz 2009).

Łomża is a city (town rights June 15, 1418 a year) with county rights situated in the north-eastern part of Poland in Podlaskie. The city is located in the middle reaches of the river Narew, which lies on the moraine hills, including a high, left-bank embankment Narew meltwater. The city is surrounded by two geographic regions - Mesopotamia Łomża and Narew Valley. In the north of the city it borders the "Rycerski Kierz" reserve and to the east of Łomża Land-

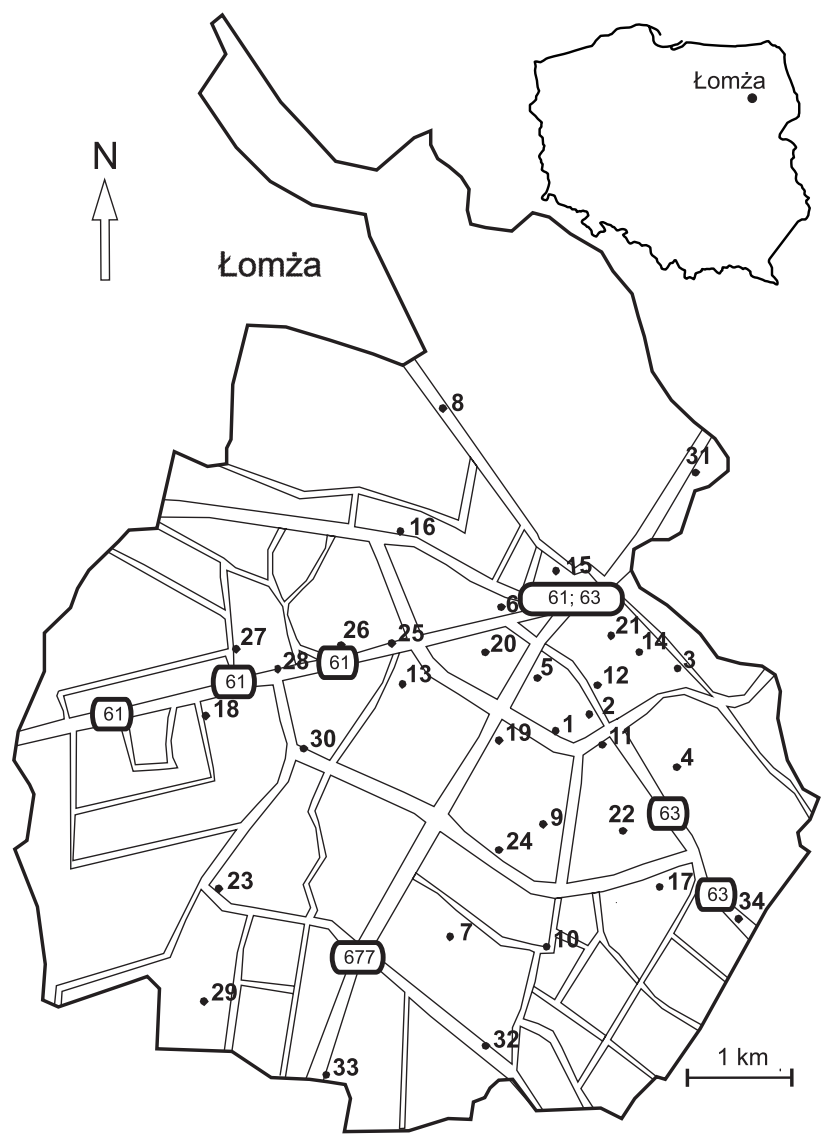

Fig. 1. Distribution of the stands in Łomża scape Park Dolina Narwi (www.lomza.polskiemiasta. info/bibliografia).

The city is located in the temperate climate zone transition. The average annual temperature is $7.1^{\circ} \mathrm{C}$, average annual rainfall of about $500 \mathrm{~mm}$. Air humidity childbearing in the range of $80-82 \%$. The growing season lasts about 200 days (GóRNIAK 2000).

The green areas located in the city occupy an area of 29.91 ha. Squares, lawns and memorials occupy an area of $12.13 \mathrm{ha}$. In addition, the city's area are three urban parks - the Jakub Waga Park, People's Park and Pope John Paul II the Pilgrim Park, which occupy an area of 17.78 ha. These areas take up $0.91 \%$ of the city. Forests, which belong to the city area occupy 17 ha are exclusively private property (JASTRZĘBSKI 2004).

The buildings are evenly spaced on, but clearly marked is the center of the city.

In 2014, it recorded 62779 residents which puts the city in this regard in 3rd place in Podlaskie (GUS 2015).

Łomża belongs to cities with a small number of large point sources of air pollution and relatively low share of emissions from these sources (Table 1). Much of the city is supplied with thermal energy (heating and hot water) from urban heating MPEC in Łomża. The main sources of air pollution in Łomża are point sources: urban heating plant MPEC, PEPEES Companies Food Industry SA. (WIOŚ 2015).

\section{MATERIALS AND METHODS}

Fieldwork research. The investigations in the area of Łomża were carried out in the years 2014-2015, on 34 research localites (Fig. 1). These stands were established within different places. The habitat was considered a stand within which the census of lichens was made from all available substrates. The epiphytic lichens were examined for individual trees with a diameter of trunks $>30 \mathrm{~cm}$. At each stand at least five trees were monitored. At each location all species of lichens on the bark of trees from the base up to $2.5 \mathrm{~m}$ were recorded.

Taxonomic identification. For most taxa, taxonomic identification was carried out in laboratory conditions. The collected specimens were determined on the basis of morphological and anatomical characters. Lichens were also determined based on the presence of secondary metabolites - test reactions. Thin-layer chromatography (TLC) was used to identify the types of secondary metabolites, mainly from the genus Cladonia, Lepraria and Rhizocarpon. This method was conducted in accordance with the guidelines from Orange et al. (2001). The species have been named according to DiEDERICH et al. (2014) and species Melanelixia to HawKsworth et al. (2008, 2011), Circinaria calcarea to Nordin et al. (2010) and Calogaya decipiens, C. pusilla, Flavoplaca citrina, F. oasis, Polycauliona polycarpa, Rusavskia elegans to ARUP et al. 
(2013). The lichen material has been deposited at the Herbarium of the Institute of Biology, University of Bialystok.

\section{RESULTS}

As a result of the study on the territory of Łomża 70 lichen species of 32 genera were recorded. The most abundant numbers of species represented here are those of genera Lecanora (17), Physcia (6) and Ramalina (3).

Lichens occur on following substrata likely to be colonized - on the bark of deciduous and coniferous trees and shrubs, wooden constructions, soil, stones, concrete, mortar, plaster and bryophytes. Lichens are represented by all morphological forms. In the biota of the investigated area, the most dominant lichens are the ones which form different types of crustose thalli. They make up 55\% of the overall number of the species. They can be found in shaded and moist places. Amongst the crustose lichens there is a significant number of pioneer species colonizing anthropogenic rock substrata, smooth tree bark and freshly-exposed soil. The second largest group consists of foliose lichens $(25 \%)$. Lichens with fruticose thalli are the less numerous group comprising $7 \%$ of the overall number of biota. The participation of lichens from other morphological groups is insignificant and amounts to around 3-6\%.

Corticolous species. The bark of trees (42 species) constitutes a substratum for numerous lichens. Out of 70 lichen species recorded in the area under study, 39 grew on tree bark including 25 obligatory epiphytes. Roadside trees dominate in Łomża, free-standing trees prevail in agricultural landscape. The richest lichen biota has been recorded on the bark of deciduous trees - Acer platanoides (26 species), Tilia cordata (28), Fraxinus excelsior (24), Tilia plathyphyllos (11). The epiphytic biota of the maple is of greater interest. Rare taxa have been identified on its bark, such as Ramalina farinacea, $R$. fastigiata.

On most roadside trees, high participation in lichen biota is exhibited by nitrophilous macrolichens of genera Physcia (P. adscendens, P. dubia, P. stellaris, P. tenella), Physconia (P. enteroxantha, P. grisea), Polycauliona polycarpa and Xanthoria parietina.

The largest amount of stands and the highest coverage coefficients are attained by species which colonize not only tree bark but other types of substrata as well. These species also comprise the most frequently encountered group in the lichen biota of Łomża: Phaeophyscia orbicularis, Physcia adscendens, Xanthoria parietina.

The rare species worth mentioning here include Ramalina fastigiata (localities: 8, 33). This species is strictly protected and is on the red list of lichens in Poland in the endangered category.
The distribution of epiphytic lichens on the territory of Łomża is uneven, which is connected with the fact that many streets and much wasteland are completely deprived of trees.

Epilithic species. The second largest habitat group - 34 species, comprises rock lichens, of which 19 are exclusive epilythes. They colonize both natural and anthropogenic substrata. Stones can be found in the open area, agricultural landscape, as well as within the town and built-up areas (walls, underpinnings and gravestones). Obligatory saxicolous species include, among others Acarospora fuscata and Rhizocarpon lavatum of which the second species is very rare in lowland Poland). Rich lichen biota occurs also on antropogenic substrata with properties resembling those of rocks, such as concrete, mortar and bricks. These become colonized by calciphilous species and also by species tolerant towards the presence of calcium carbonate, such as Calogaya decipiens, C. pusilla, Caloplaca teicholyta, Circinaria calcarea, Flavoplaca citrina, F. oasis, Lecanora albescens, L. dispersa and Xanthoria parietina. They are accompanied by numerous nitrophilous species of family Physciaceae.

Terricolous species. Soil is colonized by one lichen species of genera Cladonia - C. arbuscula.

Lignicolous species are represented only by 15 species. On the territory of Łomża lichens of dead and rotting wood favour mainly man-made timber constructions (fences, poles, crosses and farm outbuildings). The exclusive species are Lecanora varia and Micarea denigrata.

\section{PARTICIPATION OF VULNERABLE AND PROTECTED LICHENS}

Of the 70 lichen species identified in Łomża, seven species have been put on the Red list of the lichens in Poland (CIEŚLIŃsKI et al. 2006), including Rhizocarpon lavatum in critically endangered - CR, Ramalina fastigiata, $R$. fraxinea in the endangered category - EN, Ramalina farinacea in the vulnerable category - VU and Evernia prunastri, Hypogymnia tubulosa, Physcia aipolia in the category of near threatened - NT (Table 2).

Table 2. The lichen species of Łomża included in the red list of lichens in Poland

\begin{tabular}{clc}
\hline $\begin{array}{c}\text { Category } \\
\text { of threat }\end{array}$ & \multicolumn{1}{c}{ Species } & $\begin{array}{c}\text { Number } \\
\text { (percentages) } \\
\text { of species }\end{array}$ \\
\hline CR & Rhizocarpon lavatum & $1(1.5)$ \\
EN & Ramalina fastigiata, $R$. fraxinea & $2(3)$ \\
VU & Ramalina farinacea & $1(1.5)$ \\
NT & $\begin{array}{l}\text { Evernia prunastri, Hypogymnia tubulosa, } \\
\text { Physcia aipolia }\end{array}$ & $3(4)$ \\
\hline
\end{tabular}

Explanations: CR - critically endangered, EN - endangered, VU vulnerable, NT - near threatened. 
Table 3. The list of protected species occurring in Łomża

\begin{tabular}{ccc}
\hline $\begin{array}{c}\text { Status of } \\
\text { protec- } \\
\text { tion }\end{array}$ & \multicolumn{1}{c}{ Species } & $\begin{array}{c}\text { Number } \\
\text { (percentages) } \\
\text { of species }\end{array}$ \\
\hline SP & $\begin{array}{l}\text { Ramalina fastigiata, R. fraxinea } \\
\text { PP }\end{array}$ & $\begin{array}{l}\text { Cladonia arbuscula, Hypogymnia tubulo- } \\
\text { sa, Ramalina farinacea }\end{array}$ \\
\hline
\end{tabular}

Explanations: SP - strict protection, PP - partial protection.

Of all the 70 lichen species of Łomża, five have been put under legal protection, two of which are strictly and three of which are partially protected (Regulation... 2014, Table 3).

\section{LIST OF SPECIES}

Acarospora fuscata (Schrad.) Th. Fr. - stone gravestones, concrete gravestones; localities: 3, 4 .

Buellia punctata (Hoffm.) A. Massal. - bark of Aesculus hippocastanum, Acer platanoides, A. pseudoplatanus, A. saccharinum, Fraxinus excelsior, Quercus robur, Q. rubra, Robinia pseudoacacia, Sorbus aucuparia, S. intermedia, Tilia cordata, T. platyphyllos, Ulmus laevis, wooden bench, concrete gravestones; localities: 1, 4, 5, 8-11, 17, 18, 24, 26, 27, 32.

Calogaya decipiens (Arnold) Arup, Frödén \& Søchting - wooden bench, concrete (cross, wall, pole, curb, sett, board, manhole), stone wall, brick wall, grout, terrazzo; localities: $1,6,7,9,11-15$, 18-22, 24, 25, 28.

Calogaya pusilla (A. Massal.) Arup, Frödén \& Søchting - concrete (post, cross, wall, pole, curb, sett), stone gravestones, terrazzo, tombstone, stone, asbestic tile; localities: $1,3,7,9-13,15,17-20$, 22-28, 30-32.

Caloplaca teicholyta (Ach.) J. Steiner - concrete; localities: 11, 15.

Candelaria concolor (Dicks.) Arnold - bark of: Acer platanoides, Betula pendula, dead Quercus rubra; localities: 6, 34.

Candelariella aurella (Hoffm.) Zahlbr. - bryophytes, wooden bench, concrete (cross, gravestones, pole, tombstone, sett, curb, manhole, board, wall), stone, stone wall, terrazzo, grout, bricks; localities: 1, 4, 6-13, 15, 17-20, 22-26, 28, 30-32, 34.

Candelariella reflexa (Nyl.) Lettau - wooden fence; locality: 13.

Candelariella vitellina (Hoffm.) Müll. Arg. - wooden bench, stone gravestones, concrete (gravestones, curb); localities: 3, 4, 7, 12 .

Candelariella xanthostigma (Pers.) ex Ach. - bark of Acer platanoides, A. saccharinum, Betula pendula, Cupressus nootketensis, Fagus sylvatica, Fraxinus excelsior, Padus avium, Pinus sylvestris, Populus tacamahaca, P. tremula, Quercus robur, Q. rubra, Robinia pseudoacacia, Salix alba, Sorbus domestica, Tilia cordata, T. platyphyllos, dead tree, wooden bench, metal (grille, screw); localities: 1-3, 5, 6, 8, 9, 11-15, 17-19, 21, 24, 26, 27, 29, 32, 34.

Circinaria calcarea (L.) A. Nordin, Savić \& Tibell concrete gravestones; locality: 4.

Cladonia arbuscula (Wallr.) Flot. - soil in granite tombstone; locality: 1.

Cladonia coniocraea (Flörke) Spreng., nom. cons. concrete gravestones; localities: 4, 29.

Cladonia fimbriata (L.) Fr. - falled tree trunk, concrete gravestones; locality: 4 .

Evernia prunastri (L.) Ach. - bark of Acer platanoides, A. saccharinum, Betula pendula, Fraxinus excelsior, Populus tremula, Tilia cordata, T. platyphyllos, Quercus rubra; localities: 1, 3, 6, 8, 9, 11, 15, 17, 24, 34 .

Flavoplaca citrina (Hoffm.) Arup, Frödén \& Søchting - wood (fence, bench), concrete (posts, wall, gravestones, slab, board, curb, foundation), grout, stone wall, brick wall, metal structures; localities: 1, 3, 4, 7, 11-15, 17-22, 24, 25, 28.

Flavoplaca oasis (A. Massal.) Arup, Frödén \& Søchting - concrete (pole, posts, gravestones), tombstone; localities: 1, 4, 7, 11, 32.

Hypocenomyce scalaris (Ach.) M. Choisy - bark of Aesculus hippocastanum, Acer platanoides, Tilia cordata; localities: 1, 8, 27.

Hypogymnia physodes (L.) Nyl. - bark of Acer platanoides, A. saccharinum, Betula pendula, Catalpa bignonioides 'Aurea', Fraxinus excelsior, Picea abies, Pinus sylvestris, Quercus robur, Sorbus aucuparia, S. intermedia, Tilia cordata, T. platyphyllos, Ulmus laevis, wooden bench, concrete wall, gravestones; localities: 3, 6-12, 14-16, 17, 20-22, 24, 26, 27, 29, 34.

Hypogymnia tubulosa (Schaer.) Hav. - bark of Tilia cordata; locality: 17.

Lecanora albescens (Hoffm.) Flörke - concrete (post, cross, wall, gravestones, pole, slab, board, manhole), brick, brick wall, grout; localities: $1,4,6,8$, 9, 11-15, 18, 19, 20, 23-28, 30, 31.

Lecanora allophana Nyl. - bark of Acer platanoides, Tilia cordata; localities: 1, 17, 18.

Lecanora argentata (Ach.) Malme - bark of Fraxinus excelsior; locality: 8.

Lecanora carpinea (L.) Vain. - bark of Aesculus hippocastanum, Acer platanoides, Catalpa bignonioides 'Aurea', Fraxinus excelsior, Tilia cordata, Quercus rubra; localities: 1, 8, 9, 15, 26, 32.

Lecanora chlarotera Nyl. - bark of Acer negundo, A. saccharinum, Sorbus aucuparia, Tilia cordata; localities: 13, 17, 22.

Lecanora crenulata Hook. - concrete (pole); locality: 18.

Lecanora dispersa (Pers.) Sommerf. - wooden bench, concrete (pole, cross, gravestones, curb, sett, wall, foundation), brick, tombstone, stone; localities: $1,4,6-8,10,13-15,17-20,22-25,27,30,31,34$.

Lecanora expallens Ach. - bark of Acer saccharinum, Fraxinus excelsior, Tilia cordata, bryophytes; localities: 8, 24, 33. 
Lecanora hagenii (Ach.) Ach. - bark of Acer platanoides, A. saccharinum, Populus tremula, Sorbus aucuparia; localities: 2, 8, 17, 24.

Lecanora muralis (Schreb.) Rabenh. - concrete (gravestones, pole, board, posts, foundation), stone, stone gravestones, asbestic tile, sett, brick, brick wall, metal grille; localities: $1,3,4,6,7,9$, 11-15, 17-28, 30-32, 34.

Lecanora persimilis (Th. Fr.) Nyl. - concrete (pole); locality: 13.

Lecanora polytropa (Hoffm.) Rabenh. - concrete; locality: 20.

Lecanora pulicaris (Pers.) Ach. - bark of Aesculus hippocastanum, Acer platanoides, A. pseudoplatanus, A. accharinum, Fraxinus excelsior, Populus tremula, Quercus rubra, Robinia pseudoacacia, Sorbus aucuparia, S. intermedia, Tilia cordata, Ulmus laevis; localities: 1, 3, 8, 9, 15, 17, 18, 22, 34.

Lecanora rupicola (L.) Zahlbr. - gravestones, concrete gravestones; localities: 3, 4.

Lecanora saligna (Schrad.) Zahlbr. - bark of Acer platanoides; locality: 8.

Lecanora symmicta (Ach.) Ach. - bark of Tilia cordata; locality: 17.

Lecanora varia (Hoffm.) Ach. - wooden cross, wooden bench; localities: 7,8 .

Lecidella elaeochroma (Ach.) M. Choisy - bark of Aesculus hippocastanum, Acer platanoides, A. pseudoplatanus A. saccharinum, Sorbus aucuparia, Tilia cordata; localities: 1, 2, 8, 9, 13, 17, 22, 24.

Lecidella stigmatea (Ach.) Hertel \& Leuckert - concrete (cross, gravestones, pole, wall, curb, posts), brick, grout; localities: 1, 4, 6, 8-10, 13-15, 1722, 24-28, 32.

Lepraria finkii (B. de Lesd.) R.C. Harris - bark of Acer platanoides, Fraxinus excelsior, Pinus sylvestris, Tilia cordata; localities: 1, 6, 27, 29.

Lepraria incana (L.) Ach. - bark of Acer platanoides; locality: 8.

Melanelixia fuliginosa (Duby) O. Blanco, A. Crespo, Divakar, Essl., D. Hawksw. \& Lumbsch - bark of Ulmus laevis; locality: 17.

Melanelixia glabratula (Lamy) Sandler \& Arup - bark of Tilia cordata; locality: 17.

Melanohalea exasperatula (Nyl.) O. Blanco, A. Crespo, Divakar, Essl., D. Hawksw. \& Lumbsch - bark of Aesculus hippocastanum, Acer platanoides, Fraxinus excelsior, Sorbus aucuparia, S. intermedia, Tilia cordata, T. platyphyllos; localities: 8, 9, 16, 22, 24, 32-34.

Micarea denigrata (Fr.) Hedl. - wooden bench; locality: 7 .

Parmelia sulcata Taylor - bark of Acer platanoides, A. saccharinum, Betula pendula, Cupressus nootketensis, Fagus sylvatica, Fraxinus excelsior, Padus avium, Picea abies, Quercus robur, Rhus typhina, Sorbus intermedia, Tilia cordata, T. platyphyllos, Ulmus laevis, dead Quercus rubra, wooden bench, concrete; localities: 2, 5-12, 14, 15, 17, 20-22, 24, 27, 32-34.
Phaeophyscia nigricans (Flörke) Moberg - bryophytes, concrete; locality: 20.

Phaeophyscia orbicularis (Neck.) Moberg - bark of Acer negundo, A. platanoides, A. pseudoplatanus, A. accharinum, Aesculus hippocastanum, Betula pendula, Carpinus betulus, Fagus sylvatica, Fraxinus excelsior, Larix decidua, Malus sylvestris, Picea abies, Populus nigra, P. tacamahaca, P. tremula, Quercus robur, Q. rubra, Robinia pseudoacacia, Salix alba, Sorbus domestica, S. intermedia, Symphoricarpus albus, Syringa vulgaris, Thuja occidentalis, Tilia cordata, Ulmus laevis, wood (bench, fence), stone, stone wall, granite tombstone, concrete (cross, wall, gravestones, board), grout, metal, metal manhole; localities: $1-28,31-34$.

Phlyctis argena (Spreng.) Flot. - bark of Acer platanoides, Cupressus nootketensis, Fagus sylvatica, Fraxinus excelsior, Sorbus intermedia, Tilia cordata, T. platyphyllos; localities: 1, 2, 6, 8, 15, 18, 33 .

Physcia adscendens $\mathrm{H}$. Olivier, nom. cons. - bark of Acer platanoides, Acer saccharinum, Betula pendula, Catalpa bignonioides 'Aurea', Fagus sylvatica, Fraxinus excelsior, Larix decidua, Malus sylvestris, Populus tremula, Quercus robur, Q. rubra, Sorbus intermedia, Symphoricarpus albus, Tilia cordata, concrete (cross, gravestones, wall, foundation), metal; localities: 1, 4-6, 8-12, 14-22, 24, 27, 32-34.

Physcia aipolia (Humb.) Fürnr. - bark of Tilia cordata; locality: 14.

Physcia caesia (Hoffm.) Fürnr. - concrete (pole); locality: 13

Physcia dubia (Hoffm.) Lettau var. dubia - bark of Abies concolor, Acer platanoides, A. saccharinum, Aesculus hippocastanum, Betula pendula, Crataegus monogyna, Cupressus nootketensis, Fagus sylvatica, Fraxinus excelsior, F. pennsylvanica, Larix decidua, Padus avium, Pinus nigra, Populus tacamahaca, P. tremula, Quercus robur, Q. rubra, Rhus typhina, Salix alba, Sorbus domestica, S. intermedia, Tilia cordata, T. platyphyllos, dead Quercurs rubra, wooden fence, stone; localities: 1-3, 5-7, 9-24, 32-34.

Physcia stellaris (L.) Nyl. - bark of Acer platanoides, A. saccharinum, Fraxinus excelsior, Larix decidua, Malus sylvestris, Quercus robur, Q. rubra, Robinia pseudoacacia, Salix alba, Sorbus aucuparia, S. intermedia, Tilia cordata, Ulmus laevis, dead Sorbus intermedia; localities: 8-12, 17, 19, 22, 24, 34.

Physcia tenella (Scop.) DC. - bark of Acer platanoides, Betula pendula, Catalpa bignonioides 'Aurea', Fraxinus pennsylvanica, Rhus typhina, Tilia cordata, T. platyphyllos, stone, concrete (foundation, wall, curb); localities: 1, 2, 7, 8, 10, 11, 15, 17, 18, 20-22, 27, 32.

Physconia enteroxantha (Nyl.) Poelt - bark of Tilia cordata; locality: 8.

Physconia grisea (Lam.) Poelt - bark of Acer negundo, A. platanoides, Cupressus nootketensis, Fraxinus excelsior, Populus tremula, Rhus typhina, Robinia pseudoacacia, Salix alba, Sorbus domestica, S. intermedia, Tilia 
cordata, Quercus robur, falled tree trunk, concrete (post); localities: 1, 2, 5, 6, 8-12, 16-19, 24.

Polycauliona polycarpa (Hoffm.) Frödén, Arup \& Søchting - bark of Aesculus hippocastanum, Acer saccharinum, Betula pendula, Catalpa bignonioides 'Aurea', Fraxinus excelsior, Malus sylvestris, Picea abies, Populus nigra, Quercus robur, Q. rubra, Rhus typhina, Robinia pseudoacacia, Sorbus aucuparia, S. intermedia, dead Sorbus intermedia, wooden bench; localities: 6-11, 14-20, 22, 26, 27, 34.

Pseudevernia furfuracea (L.) Zopf - bark of Fraxinus excelsior; locality: 8.

Ramalina farinacea (L.) Ach. - bark of Fraxinus excelsior; locality: 8.

Ramalina fastigiata (Pers.) Ach. - bark of Fraxinus excelsior; localities: 8, 33.

Ramalina fraxinea (L.) Ach. - bark of Acer platanoides, Fraxinus excelsior, Sorbus intermedia, Tilia cordata; localities: 8, 17, 33 .

Rhizocarpon lavatum (Fr.) Hazsl. - stone gravestones; localities: 3, 4.

Rusavskia elegans (Link) S.Y. Kondr. \& Kärnefelt concrete (pole, poles, foundation, curb); localities: 9, 13, 18, 20, 22, 24, 26, 30.

Sarcogyne regularis Körb. - concrete (wall); locality: 15.

Scoliciosporum chlorococcum (Stenh.) Vězda - bark of Acer platanoides, Betula pendula; localities: 5, 6 .

Scoliciosporum umbrinum (Ach.) Arnold - stone, concrete; localities: 1, 3, 20.

Verrucaria muralis Ach. - concrete wall; locality: 15.

Verrucaria nigrescens Pers. - concrete (posts, gravestones, wall, foundation, manhole); localities: 1 , 4, 9, 11, 13-15, 18, 19, 21, 24, 26, 28, 32.

Xanthoria parietina (L.) Th. Fr. - bark of Acer negundo, A. platanoides, A. pseudoplatanus, A. saccharinum, Aesculus hippocastanum, Betula pubescens, Catalpa bignonioides 'Aurea', Crataegus monogyna, Fagus sylvatica, Fraxinus excelsior, F. pennsylvanica, Larix decidua, Malus sylvestris, Pinus nigra, Platanus acerifolia, Populus nigra, P. tacamahaca, P. tremula, Quercus robur, Q. rubra, Rhus typhina, Robinia pseudoacacia, Salix alba, Sambucus nigra, Sorbus domestica, S. intermedia, Symphoricarpus albus, Syringa vulgaris, Thuja occidentalis, Tilia cordata, T. platyphyllos, Ulmus laevis, dead Sorbus intermedia, wooden bench, concrete (wall, gravestones, poles, foundation) granite tombstone, stone, metal fence; localities: 1-3, 5-34.

\section{LIST OF LOCALITIES}

Mikołaj Kopernik Street, Cathedral cemetery, located in city center, the oldest cemetery in Łomża city, opened in 1801 , monument $\left(53^{\circ} 10^{\prime} 22.8^{\prime \prime} \mathrm{N}\right.$, $\left.22^{\circ} 04^{\prime} 29.8^{\prime \prime} \mathrm{E}\right)$ - Acer platanoides, Fraxinus excelsior, Tilia cordata, constructions of: metal, stone and concrete (headstones, crosses, foundations, fence).
Mikołaj Kopernik Street, square near Cathedral cemetery $\left(53^{\circ} 10^{\prime} 26.2^{\prime \prime} \mathrm{N}, 22^{\circ} 04^{\prime} 39.2^{\prime \prime} \mathrm{E}\right)$ - Acer negundo, A. platanoides, A. saccharinum, Betula sp., Fraxinus pennsylvanica, Populus tremula, Rhus typhina, Robinia pseudoacacia, Tilia platyphyllos, concrete wall.

Rybaki Street, Jewish cemetery, located in the eastern part of the city $\left(53^{\circ} 10^{\prime} 38.2^{\prime \prime} \mathrm{N}, 22^{\circ} 05^{\prime} 17.8^{\prime \prime} \mathrm{E}\right)$ - Populus tremula, stone, gravestones,

Boczna Street, Jewish cemetery, located in district detached houses $\left(53^{\circ} 10^{\prime} 17.4^{\prime \prime} \mathrm{N}, 22^{\circ} 05^{\prime} 21.5^{\prime \prime} \mathrm{E}\right)$ - concrete steles, stone, gravestones, brick,

Avenue of Legions, "Park Ludowy", located near provincial road number 677, founded in 1905 $\left(53^{\circ} 10^{\prime} 30.9^{\prime \prime} \mathrm{N}, 22^{\circ} 04^{\prime} 16.5^{\prime \prime} \mathrm{E}\right)$ - Acer platanoides, A. pseudoplatanus, Aesculus hippocastanum, Carpinus betulus, Fagus sylvatica, Fraxinus excelsior, Pinus nigra, Quercus robur (natural monument), Q. rubra, Robinia pseudoacacia, Salix alba.

Wojska Polskiego Street, Park named of the Jakub Waga, located near national road number 63 - including international transit route, founded in 1842 , in the central part is a Jakub Waga monument $\left(53^{\circ} 10^{\prime} 50.1^{\prime \prime} \mathrm{N}, 22^{\circ} 04^{\prime} 11.4^{\prime \prime} \mathrm{E}\right)$ - Abies concolor, Acer negundo, A. platanoides, A. pseudoplatanus, Betula pendula, Fagus sylvatica (natural monument), Fraxinus excelsior, Padus avium, Populus tremula, Quercus rubra, Salix alba, Syringa vulgaris, Thuja sp., Tilia cordata, Ulmus laevis, wood.

Przykoszarowa Street, Catholic municipal cemetery, located in the southern part of the city

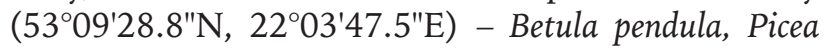
abies, wooden constructions (cross 1985, bench), granite tombstones, headstones material terrazzo, sett, concrete structures (curb, wall), grout, brick.

Grobla Jednaczewska Street, located in northern part of the city, on the border with reserve "Rycerski Kierz", locality from the bridge on Łomżyczka to allotments $\left(53^{\circ} 11^{\prime} 40.7^{\prime \prime} \mathrm{N}, 22^{\circ} 03^{\prime} 44.0^{\prime \prime} \mathrm{E}\right)$ - Acer platanoides, Fraxinus excelsior, Salix alba, Tilia cordata, T. platyphyllos, concrete, brick, wooden bench, metal poles.

Pope John Paul II the Pilgrim Park (530.'56.5"N, $\left.22^{\circ} 04^{\prime} 24.8^{\prime \prime} \mathrm{E}\right)$ - Acer platanoides, Aesculus hippocastanum, Betula pendula, Malus sylvestris, Populus tremula, Robinia pseudoacacia, Tilia cordata, Quercus rubra, concrete structures (wall, lighting pole).

Julian Ursyn Niemcewicz Street, near apartment building number $5\left(53^{\circ} 09^{\prime} 27.6^{\prime \prime} \mathrm{N}, 22^{\circ} 04^{\prime} 25.2^{\prime \prime} \mathrm{E}\right)$ Betula pendula, Larix decidua, Rhus typhina, Robinia pseudoacacia, Tilia cordata, concrete curb.

Władysław Sikorski Street, locality from the Lutosławski roundabout to the Zawadzka junction of the street $\left(53^{\circ} 10^{\prime} 17.3^{\prime \prime} \mathrm{N}, 22^{\circ} 04^{\prime} 50.8^{\prime \prime} \mathrm{E}\right)$ - Acer platanoides, Sorbus domestica, Tilia cordata, wooden structures (pole, bench), concrete structures (posts, sewer drain, wall), brick wall, grout.

Niepodległości Square, square opposite "Lalki i Aktora" Theatre $\left(53^{\circ} 10^{\prime} 29.2^{\prime \prime} \mathrm{N}, 22^{\circ} 04^{\prime} 45.6^{\prime \prime} \mathrm{E}\right)$ - Acer 
platanoides, Populus tremula, Salix alba, Sorbus domestica, Tilia platyphyllos, concrete structures (slab, board, cur), metal structures (lighting poles, manhole).

Fabryczna Street, around the production plant

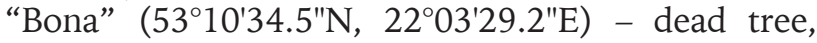
wooden structure (fence, pole), concrete structures (pole, curb, wall, manhole, posts).

Senatorska 13 Street, High School number 13, located near town hall $\left(53^{\circ} 10^{\prime} 39.7^{\prime \prime} \mathrm{N}, 22^{\circ} 05^{\prime} 04.7^{\prime \prime} \mathrm{E}\right)$ - Acer platanoides, Betula pendula, Quercus rubra, Tilia cordata, wooden constructions (bench), concrete structures.

Piękna Street $\left(53^{\circ} 10^{\prime} 59.1^{\prime \prime} \mathrm{N}, 22^{\circ} 04^{\prime} 27.0^{\prime \prime} \mathrm{E}\right)$ - Acer platanoides, Aesculus hippocastanum, Betula pendula, Larix decidua, Sorbus intermedia, Symphocarpos albus, Tilia cordata, wooden structure (wall, pole), stone constructions (wall), grout.

Nowogrodzka 60 Street, Forestry Offices of Łomża, located near locality number $6\left(53^{\circ} 11^{\prime} 02.3^{\prime \prime} \mathrm{N}\right.$, $\left.22^{\circ} 03^{\prime} 46.3^{\prime \prime} \mathrm{E}\right)$ - Sorbus intermedia.

Marszałek Józef Piłsudski Street, Provincial Hospital area $\left(53^{\circ} 09^{\prime} 39.6^{\prime \prime} \mathrm{N}, 22^{\circ} 05^{\prime} 14.7^{\prime \prime} \mathrm{E}\right)$ - Acer platanoides, Sorbus intermedia, Tilia cordata, dead Sorbus intermedia, concrete constructions (wall, slab), metal grille.

Wojska Polskiego Street, from Ciepła Street to Studencka Street, located on National road number 61 (53 $\left.{ }^{\circ} 10^{\prime} 29.2^{\prime \prime} \mathrm{N}, 22^{\circ} 02^{\prime} 08.5^{\prime \prime} \mathrm{E}\right)$ - Acer platanoides, Fraxinus excelsior, Tilia cordata, dead tree trunk, concrete structures (foundation, the basics of advertising banners).

Adam Mickiewicz Street, located near Primary school number $7\left(53^{\circ} 10^{\prime} 15.5^{\prime \prime} \mathrm{N}, 22^{\circ} 04^{\prime} 06.0^{\prime \prime} \mathrm{E}\right)$ - Quercus robur, concrete constructions (slab, pole, wall).

Kaktusowa Street $\left(53^{\circ} 10^{\prime} 41.4^{\prime \prime} \mathrm{N}, 22^{\circ} 03^{\prime} 53.4^{\prime \prime} \mathrm{E}\right)$ Quercus robur, concrete constructions (pole, curb).

Farna Street $\left(53^{\circ} 10^{\prime} 43.0^{\prime \prime} \mathrm{N}, 22^{\circ} 04^{\prime} 48.9^{\prime \prime} \mathrm{E}\right)$ - Tilia cordata, T. platyphyllos, concrete constructions (wall).

Księżna Anna Street, located in sports and recreation complex $\left(53^{\circ} 09^{\prime} 57.1^{\prime \prime} \mathrm{N}, 22^{\circ} 04^{\prime} 59.2^{\prime \prime} \mathrm{E}\right)$ - Acer saccharinum, Betula pendula, Picea abies, Sorbus aucuparia, $S$. intermedia, concrete structures (foundation, pole).

Poznańska 148 Street, located near Browar Łomża $\left(53^{\circ} 09^{\prime} 33.4^{\prime \prime} \mathrm{N}, 22^{\circ} 02^{\prime} 11.6^{\prime \prime} \mathrm{E}\right)$ - concrete structures (poles), metal constructions (poles).

Józef Bem Street, from Władysław Broniewski Street to Władysław Reymont Street (530.' $51.9^{\prime \prime} \mathrm{N}$, $22^{\circ} 03^{\prime} 53.4^{\prime \prime E)}$ - Acer saccharinum, Sorbus intermedia, Tilia cordata, T. platyphyllos, wooden structures (decorative fence), concrete structures (lighting poles, curb, posts).

Kręta Street - Pinus sylvestris, Rhus typhina, concrete structures (curb, pole, wall), sett $\left(53^{\circ} 10^{\prime} 42.7^{\prime \prime} \mathrm{N}\right.$, $\left.22^{\circ} 03^{\prime} 22.4^{\prime \prime} \mathrm{E}\right)$.

Janusz Korczak Street $\left(53^{\circ} 10^{\prime} 40.2^{\prime \prime} \mathrm{N}, 22^{\circ} 03^{\prime} 08.3^{\prime \prime} \mathrm{E}\right)$ - Betula pendula, Fraxinus excelsior, Rhus typhina, con- crete structures (pole, posts, wall), metal structures (fence, screw),

Piaski Street, from Świerkowa street to Podleśna street, locality located on "Maria" residential $\left(53^{\circ} 10^{\prime} 42.5^{\prime \prime} \mathrm{N}, 22^{\circ} 02^{\prime} 12.2^{\prime \prime} \mathrm{E}\right)$ - Tilia cordata, wooden structure (pole), concrete structures (wall, pole), metal structure (fence).

Piwna Street $\left(53^{\circ} 10^{\prime} 36.5^{\prime \prime} \mathrm{N}, 22^{\circ} 02^{\prime} 37.2^{\prime \prime} \mathrm{E}\right)$ - Salix alba, concrete structures (poles, manhole), eternity tiles.

Poligonowa Street $\left(53^{\circ} 08^{\prime} 55.8^{\prime \prime} \mathrm{N}, 22^{\circ} 02^{\prime} 13.5^{\prime \prime} \mathrm{E}\right)-$ Pinus sylvestris, Sambucus nigra, felled tree trunk.

Marszałek Józef Piłsudski Street, from Poznańska Street to Spokojna Street $\left(53^{\circ} 10^{\prime} 11.3^{\prime \prime N}\right.$, $\left.22^{\circ} 02^{\prime} 48.2^{\prime \prime} \mathrm{E}\right)$ - wooden structures (post), concrete structure (pole).

Zjazd Street, locality on the border with Piątnica $\left(53^{\circ} 11^{\prime} 24.4^{\prime \prime} \mathrm{N}, 22^{\circ} 05^{\prime} 24.2^{\prime \prime} \mathrm{E}\right)$ - Tilia cordata, concrete structures (poles).

Highway to Mężenin (5309'08.1"N, 2203'37.6"E) - Acer pseudoplatanus, Tilia cordata, Picea abies, concrete structure (fence).

Avenue of Legions, locality on the border with Konarzyce $\left(53^{\circ} 08^{\prime} 59.0^{\prime \prime} \mathrm{N}, 22^{\circ} 02^{\prime} 53.3^{\prime \prime} \mathrm{E}\right)$ - Fraxinus excelsior.

Szosa Zambrowska Street $\left(53^{\circ} 09^{\prime} 46.1^{\prime \prime} \mathrm{N}\right.$, $\left.22^{\circ} 05^{\prime} 30.9^{\prime \prime} \mathrm{E}\right)$ - Acer platanoides, Fraxinus excelsior, Tilia cordata, concrete structures (curb, posts).

\section{DISCUSSION}

The lichen biota of Łomża consists of 70 species of lichens. This number is relatively small considering the results of research conducted in a similar type of cities in other parts of the country. In Poland, it has been studied more than 50 cities (MATwiejuK \& KorobKIEWICZ 2012b). A similar number of species recorded in Lublin - 73 (RYDZAK 1953), Międzyzdroje - 68 (IZYDOREK 2005) and Świnoujście - 74 (IZYDOREK 2005). The greatest number of species recorded in Olsztyn - 251 (Kubiak 2005). In north-eastern Poland, in Narew 97 species of lichens were observed (Matwiejuk \& Korobkiewicz 2012a), in Mielnik - 91 (Matwiejuk 2008), in Drohiczyn - 86 (Matwiejuk 2009b) (Table 4).

The small number of lichen species in Łomża can be conditioned by the absence of forest complexes in the city. The lichen biota of Łomża have been not recorded species lichens characteristic for forest areas. For comparison the city of Olsztyn area is characterized, as compared to other Polish cities, by a much higher overall number of lichens taxa, higher number of rare, protected and threatened species as well as a lower number of locally rare taxa. In Olsztyn, more than $21 \%$ of the city area is taken by the municipal forest. The indicators of lowland old-growth forests, to a limited extent, is one of the most characteris- 
Table 4. The summarizes of the number of species of lichens in cities in the Podlasie voivodeship similar size to Łomża based on literature and own data

\begin{tabular}{|c|c|c|c|c|c|c|c|c|}
\hline \multirow{2}{*}{ City } & \multirow{2}{*}{ Literature } & \multicolumn{7}{|c|}{ Number of species } \\
\hline & & total & corticolous & lignicolous & saxicolous & terricolous & muscicolous & other \\
\hline Łomża & & 70 & 39 & 15 & 34 & 1 & 3 & 5 \\
\hline Supraśl & Matwiejuk (2015) & 89 & 57 & 46 & 39 & - & 7 & 9 \\
\hline Ciechanowiec & $\begin{array}{l}\text { Matwiejuk \& } \\
\text { Kolanko (2007) }\end{array}$ & 114 & 57 & 54 & 41 & 22 & 4 & 10 \\
\hline Mielnik & Matwiejuk (2008) & 91 & 47 & 26 & 38 & 13 & 2 & 1 \\
\hline Boćki & Matwiejuk (2009a) & 118 & 57 & 41 & 38 & 31 & 2 & 15 \\
\hline Drohiczyn & Matwiejuk (2009b) & 86 & 44 & 6 & 32 & 15 & - & - \\
\hline Narew & $\begin{array}{l}\text { Matwiejuk \& Ko- } \\
\text { robkiewicz }(2012 a)\end{array}$ & 97 & 38 & 50 & 36 & 19 & - & 20 \\
\hline
\end{tabular}

tic elements of the local biota differentiating Olsztyn from among other Polish cities (KUBIAK 2005).

Epiphytes are the most numerous habitat group in cities. The analysed towns differ due to a significant number of species growing on the bark of trees and shrubs (Białowieża - 68 species, Limanowa - 60, Supraśl, Ciechanowiec, Boćki - 57, Drohiczyn - 44, Drezdenko - 39, Narew - 38, Łomża - 39). Their rich species variety and abundant occurrence highlights the maintenance of biocenotic structures deformed to an insignificant extent by anthropopressure.

The number of epiphytes in Polish cities is comparable to the cities such as in Ukraine, and Italy. In Ukrainian and Italian cities where both suburban forests and the built-up area have been investigated and all available tree species have been monitored, only 35-60 epiphytic lichen species have been recorded, e.g. 38 species in Kherson (Khodosovtsev 1995), 48 in Lviv (KoNDRATYUK et al. 1991), 56 in Chernigiv (Zelenko 1999) 57 in Sienna (Loppi et al. 2002) and 65 in Kyiv (Dymytrova 2009).

Among the lichens colonizing secondary rock substrata the most frequently represented habitat group are calciphilous lichens bound up with concrete. These species are spread especially in city zone. The most common-place species are Lecanora albescens, L. dispersa, Calogaya decipiens, C. pusilla, Candelariella aurella, Phaeophyscia orbicularis, Xanthoria parietina. They frequently cover large surfaces, especially the old plaster of houses, walls and poles.

In Łomża, as in other parts of the country, the presence of epixylous lichens is marked on the wooden buildings, fences around fields, buildings, gardens, pillars (MATwiejuk 2008, 2009a, b). The list of this group is dominated by common lichen taxa and those exhibiting a wide range of ecological spectrum, developing both on the wood and the bark of trees and on soil.

Terricolous lichens have been found outside urban settlements, primarily in forest fragments, grassland, on sandy wasteland situated in the vicinity of the administrative boundaries of the city and on the outskirts. In all of the towns, a group of species can be differentiated, the ones which have found here optimal living conditions.

\section{ACKNOWLEDGEMENTS}

The paper has been financially supported by the Polish Ministry of Science and Higher Education through statutory research.

\section{REFERENCES}

Arup U., SøChting U., FrödÉn P. (2013): A new taxonomy of the family Teloschistaceae. Nordic Journal of Botany 31,1: 16-83.

CiEŚLIŃSKI S. (1974): Flora epifityczna porostów miasta Radom. Biuletyn Kwartalny Radomskiego Towarzystwa Naukowego 11, 3/4: 169-189.

CieśLIŃSKI S., CZYŻewsKa K., FABISZEwsKI J. (2006): Red list of the lichens in Poland. In: Z. Mirek, K. Zarzycki, W. Wojewoda, Z. Szeląg (eds). Red list of plants and fungi in Poland. W. Szafer Institute of Biology, Polish Academy of Sciences, Kraków: 71-90.

Diederich P., Ertz D., Stapper N., Sérusiaux E., Van den Broeck D., van den Boom P., Ries C. (2014): The lichens and lichenicolous fungi of Belgium, Luxembourg and northern France. URL: http:// www.lichenology.info (access: 20.09.2014).

DymYtrova L. (2009): Epiphytic lichens and bryophytes as indicators of air pollution in Kyiv city (Ukraine). Folia Cryptogamica Estonica 46: 33-44.

FAŁTYNOWicz W., IZYDOREK I., BUDZBon E. (1991): The lichen flora as bioindicator of air pollution of Gdańsk, Sopot and Gdynia. Monographiae Botanicae 73: 1-52.

GóRniAK A. (2000): Klimat województwa podlaskiego. Instytut Meteorologii i Gospodarki Wodnej, Oddział w Białymstoku.

GUS - Główny Urząd Statystyczny (2014): Ludność według płci i miast (access: 28.10.2015).

Hawksworth D.L., Blanco O., Divakar P.K., Ahti T., Crespo A. (2008): A first checklist of parmelioid and similar lichens in Europe and some adjacent 
territories, adopting revised generic circumscriptions and with indications of species distributions. Lichenologist 40(1): 1-21.

Hawksworth D.L., Divakar P.K., Crespo A., Ahti T. (2011): The checklist of parmelioid and similar lichens in Europe and some adjacent territories: additions and corrections. Lichenologist 43(6): 639-645.

IZYDOREK I. (2005): Porosty wybranych miast na polskim wybrzeżu Bałtyku. Acta Botanica Cassubica 5: $173-178$.

Jagietło M. (1983): Porosty epifityczne Limanowej. Zeszyty Naukowe Uniwersytetu Jagiellońskiego. Prace Botaniczne 11: 191-218.

JASTRZĘBSKi W. (2004): Plan rozwoju lokalnego miasta Łomża na lata 2004-2006, Łomża URL: http:// www.lomza.pl/bip/zalaczniki/art/040616104428. doc (access: 15.04.2016).

Khodosovtsev A.Ye. (1995): Lichen indication assessment of Kherson city (in Russian). Konstanty 2: $52-60$.

KiszKA J. (1977): Wpływ emisji miejskich i przemysłowych na florę porostów (Lichenes) Krakowa i Puszczy Niepołomickiej. Prace Monograficzne Wyższej Szkoły Pedagogicznej w Krakowie 19: $5-137$.

KIsZKA J. (1999): Porosty (Lichenes) oraz warunki bioekologiczne Przemyśla. Arboretum Bolestraszyce 6: 1-86.

Kondracki J. (1972): Polska Północno-Wschodnia. PWN, Warszawa.

KondRATYuK S.YA., KoucheriaVyi V.O., Kramarets V.O., ZiNKO YU.V., SiRENKO I.M. (1991): Lichen indication of air pollution in Lviv (in Ukrainian). Ukrainskyi Botanichnyi Zhurnal 48(2): 72-76.

KuBIAK D. (2005): Lichens and lichenicolous fungi of Olsztyn town (NE) Poland. Acta Mycologica 40(2): 293-332.

LAUNDON J.R. (2010): Lecanora antiqua, a new saxicolous species from Great Britain, and the nomenclature and authorship of L. albescens, L. conferta and L. muralis. Lichenologist 42, 6: 631-635.

LIPNICKI L. (1984): Porosty miasta Drezdenka i najbliższej okolicy. Fragmenta Floristica et Geobotanica 28, 2: 221-239.

Loppi S., Ivanov D., BocCardi R. (2002): Biodiversity of epiphytic lichens and air pollution in the town of Siena (Central Italy). Environmental Pollution 116(1): 123-128.

Matwiejuk A. (2007): Porosty Białegostoku. Analiza florystyczno-ekologiczna. T. 1. Wydawnictwo „Ekonomia i Środowisko”, Białystok.

Matwiejuk A. (2008): Lichens of Mielnik on river Bug (Podlasie, Eastern Poland). Nature Journal 41: 5-18.

Matwiejuk A. (2009a): Lichens of the Boćki and its surroundings in Podlasie (NE Poland). Nature Journal: 42: 49-61.
Matwiejuk A. (2009b): Lichens of Drohiczyn on the Bug river (Podlasie, Eastern Poland). Roczniki Akademii Rolniczej w Poznaniu 387, Botanika-Steciana 12: 57-62.

Matwiejuk A. (2011): Anthropogenic changes of lichen biota of the Białowieża town (Podlasie, Eastern Poland). Roczniki Akademii Rolniczej w Poznaniu 390, Botanika-Steciana 15: 129-138.

Matwiejuk A. (2015): Lichens of the Supraśl town (Podlasie, north-eastern Poland). Steciana 19(3): 133-142.

Matwiejuk A., Kolanko K. (2007): Lichens of Ciechanowiec and its environs (Eastern Poland). Roczniki Akademii Rolniczej w Poznaniu 386, Botanika-Steciana 11: 85-93.

Matwiejuk A., Korobkiewicz K. (2012a): Lichens of Narew and its surroudings (Podlasie, North-Eastern Poland). Roczniki Akademii Rolniczej w Poznaniu 391, Botanika-Steciana 16: 93-100.

Matwiejuk A., Korobriewicz K. (2012b): Stan badań bioty porostów w miastach Polski. Ochrona Środowiska i Zasobów Naturalnych 51: 85-105.

Matwiejuk A., Wójtowicz E. (2013): Porosty Ełku $\mathrm{w}$ województwie warmińsko-mazurskim. In: I. Ciereszko, A. Bajguz (eds). Różnorodność biologiczna - od komórki do ekosystemu. Rośliny i grzyby w zmieniających się warunkach środowiska. Polskie Towarzystwo Botaniczne, Białystok 21: 291-306.

Nordin A., Savić S., Tibell L. (2010): Phylogeny and taxonomy of Aspicilia and Megasporaceae. Mycologia 102: 1339-1349.

Orange A., James P.W., White FJ. (2001): Microchemical methods for the identification of lichens. British Lichen Society, London.

Pustelniak L. (1991): Epiphytic lichens of the city Rzeszów (South-Eastern Poland). Zeszyty Naukowe Uniwersytetu Jagiellońskiego Prace Botaniczne 22: 171-191.

RozPORZĄDZENIE Ministra Środowiska $z$ dnia 9 października 2014 r. w sprawie gatunków dziko występujących grzybów objętych ochroną. (2014). Dz.U. z 2014, poz. 408, z późn. zm.

RyDZAK J. (1953): Rozmieszczenie i ekologia porostów miasta Lublina. Annales Universitatis Mariae Curie-Skłodowska. Sectio C, Biologia 8, 9: 233-356.

RYDZAK J. (1956a): Wpływ małych miast na florę porostów. Part 1. Dolny Śląsk - Kluczbork-WołczynOpole-Cieszyn. Annales Universitatis Mariae Curie-Skłodowska Sectio C, Biologia 10, 1: 1-32.

RYDZAK J. (1956b): Wpływ małych miast na florę porostów. Part 2. Beskidy Zachodnie. Wisła-Ustroń-Muszyna-Iwonicz-Rymanów-Lesko. Annales Universitatis Mariae Curie-Skłodowska. Sectio C, Biologia 10, 2: 33-66.

RYDZAK J. (1957): Wpływ małych miast na florę porostów. Part 4. Lubelszczyzna-Kieleckie-Podlaskie- 
Puławy-Busko-Siedlce-Białowieża. Annales Universitatis Mariae Curie-Skłodowska. Sectio C, Biologia 10, 14: 321-398.

RYDZAK J. (1959): Wpływ małych miast na florę porostów. Part 5. Kotlina Kłodzka - Kłodzko, Kudowa-Zdrój, Duszniki-Zdrój, Polanica-Zdrój, Lądek-Zdrój, Stronie Śląskie. Annales Universitatis Mariae Curie-Skłodowska. Sectio C, Biologia 11, 2: 25-50.

ŚPIEWAKowski E.R., Izydorek I. (1981): Porosty Słupska na tle warunków ekologicznych miasta. Wyższa Szkoła Pedagogiczna, Słupsk.

Toвorowicz K. (1976): Porosty miasta Kielc i najbliższej okolicy. Fragmenta Floristica Geobotanica 22, 4: 574-603.

Urząd Miejski w Łomży. (2000): Profesjonalna Gmina. Libra-Print, Łomża. http://lomza.polskiemiasta.info/bibliografia (access: 3.03.2016).

WIOŚ - Wojewódzki Inspektorat Ochrony Środowiska w Białymstoku Delegatura w Łomży (2015):
Informacja Podlaskiego Wojewódzkiego Inspektora Ochrony Środowiska w Białymstoku o stanie środowiska na terenie Łomży w 2014 roku. WIOŚ, Łomża: 1-57.

Zelenko S.D. (1999): Assessment of air pollution in Chernigiv using epiphytic lichens (in Ukrainian). Ukrainskyi Botanichnyi Zhurnal 56(1): 64-67.

ZimnY H., KucińsKa K. (1974): Porosty Warszawy jako biowskaźniki zaburzeń środowiska miejskiego. Przegląd Informacyjny - Zieleń Miejska 10/1. Instytut Gospodarki Komunalnej, Warszawa: 13-22. ZubKowicz R. (2009): Łomża - gród na nadnarwiańskiej skarpie, Polska Turystyczna.pl., Urząd Miejski w Łomży, Kraków.

For citation: Matwiejuk A., Chojnowska P. (2016): Lichens of Łomża town (Podlasie, north-eastern Poland). Steciana 20(2): 53-62. doi: 10.12657/ steciana.020.007 